\title{
Ultra-wideband Time-delay Line Inspired by Composite Right/left-handed Transmission Line Unit Cell
}

\author{
J. Zhang, S.W. Cheung and T.I. Yuk \\ Department of Electrical and Electronic Engineering, \\ The University of Hong Kong, \\ Pokfulam Road, Hong Kong, China
}

\begin{abstract}
This paper presents a design of ultra-wideband timedelay line inspired by the composite right/left-handed transmission line (CRLH TL) unit cell. A rotated version of the conventional CRLH TL unit cell is used to increase the operating bandwidth. The time-delay line is optimized using computer simulation and then fabricated on a PCB for measurement. For comparison, the time-delay lines using the right-handed transmission line (RH TL) and periodic microstrip line (PML) are also designed, optimized and fabricated. Simulation and measurement results show that, for the same length of $60 \mathrm{~mm}$, our proposed time-delay line has a much longer time delay of 2000 ps with high return loss and low insertion loss.
\end{abstract}

\section{INTRODUCTION}

Time-delay lines have found applications in different areas, such as signal processing systems, telecommunications systems and phased array systems. For example, time-delay lines are used in the feedforward configuration for analogue predistortion of power amplifiers [1]. In the design of timedelay lines for microwave applications, the size, return loss and insertion loss are major concerns. Time-delay lines based on waveguides are low loss, but bulky and expensive, while timedelay lines based on transmission lines are of smaller sizes and losses.

The concept of metamaterials, commonely known as lefthanded materials (LHMs), was first investigated by Veselago in 1968 [2]. Metamaterials have negative permittivity and permeability which are not commonly found in nature. Although the properties of LMHs promised for a large diversity of novel applications and devices, LMHs did not attract much attention until it was found that the materials could be realized using a general transmission line (TL) approach [3]. Practical LH TL also have the right-handed (RH) effects, so LHMs realized using TLs are called composite right/left handed transmission lines (CRLH TLs). CRLH TL can be used to design many different microwave components such as phase shifters, antennas and bandpass filters, etc.

In this paper, we propose to use a rotated version of a conventional CRLH TL unit cell [4] to design a time-delay line for ultra-wideband (UWB) applications. Simulation and measurement results show that our proposed time-delay line can provide a much longer time delay than those of the timedelay lines based on the periodic microstrip line (PML) [4] and the traditional right-handed transmission line (RH TL). Moreover, it has an ultra-wide operation bandwidth of $0.1-10$ $\mathrm{GHz}$, a high return loss of more than $15 \mathrm{~dB}$ and a low insertion loss of less than $1 \mathrm{~dB}$.

\section{TIME-DELAY LINE USING TRANSMISSION LINE}

One of the important parameters for a transmission line is the time delay which describes how long it takes for a signal to travel from one end of the transmission line to the other end. This time-delay property can be used to design time-delay lines. For a transmission line with a length of $L$, the time delay for a signal to travel through is given by:

$$
t_{\text {delay }}=\frac{L}{\lambda f}=\frac{L \times \beta}{2 \pi f}
$$

where $f$ and $\beta$ are the operating frequency and the phase constant, respectively, of the transmission line. It can be seen from (1) that the longer the transmission line used, the longer is the time delay. However, it can also be seen from the same equation that a larger phase constant $\beta$ will produce a longer time delay. Or equivalently, for a given time delay, a larger $\beta$ requires a shorter transmission line. Thus, to obtain a large time delay with a short transmission line at a specific frequency, a large phase constant $\beta$ is needed.

\section{MODEL OF CRLH TL UNIT CELL}
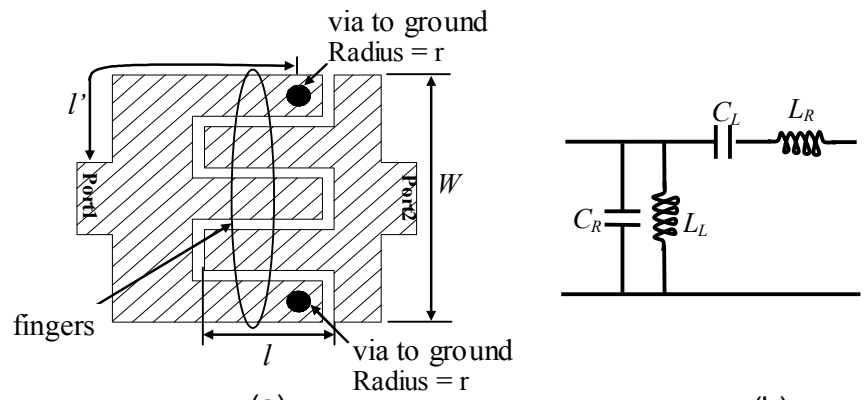

(a)

(b)

Figure 1 (a) Structure and (b) equivalent circuit of conventional CRLH TL unit cell 
The general schematic figure and the equivalent circuit of a CRLH TL unit cell [4] with five fingers are shown in Fig. 1, where in Fig. 1(b) the series inductance $L_{R}$ and capacitance $C_{L}$ are the inductance along the fingers and the coupling capacitance between the fingers, respectively, the shunt inductance $L_{L}$ is realized by the two fingers each having a via at the ends to the ground, and the shunt capacitance $C_{R}$ is the stray capacitance of the fingers. The expressions for $L_{R}, C_{R}, L_{L}$ and $C_{L}$ are, respectively, given by $[5,6]$ :

$$
\begin{gathered}
L_{R}=\frac{Z_{0} \sqrt{\varepsilon_{r e}}}{c} l \\
C_{R}=\frac{\sqrt{\varepsilon_{r e}}}{Z_{0} c} l \\
C_{L}=\left(\varepsilon_{r}+1\right) l\left\{4.409(N-3) \tanh \left[0.55\left(\frac{h}{W}\right)^{0.45}\right]\right. \\
\left.\left.\left.+9.92 \tanh \left[0.52\left(\frac{h}{W}\right)^{0.5}\right]\right\} \times 10^{-12}\right]\right) \\
L_{L}=\frac{\mu_{0}\left[h \cdot \ln \left(\frac{h+\sqrt{r^{2}+h^{2}}}{2 \pi}\right)+\frac{3}{2}\left(r-\sqrt{r^{2}+h^{2}}\right)\right]}{r}+\frac{Z_{0} \sqrt{\varepsilon_{r e}}}{c} l^{\prime}
\end{gathered}
$$

where $h$ is the thickness of the substrate, $r$ is the radius of the ground via, $\varepsilon_{r}$ is the relative dielectric constant, $\varepsilon_{r e}$ is the effective dielectric constant, $l$ ' is the distance from the ground via to the port, $l$ is the length of the finger, $N$ is the number of the fingers, $W$ is the width of all the fingers together and $Z_{0}$ is the characteristic impedance of each of the fingers. The phase constant $\beta$ of such CRLH TL unit cell is [7]:

$$
\beta=\omega \sqrt{L_{R} C_{R}}-\frac{1}{\omega \sqrt{L_{L} C_{L}}}
$$

(2) - (6) show that the structural parameters $L_{R}, C_{R}, L_{L}$ and $C_{L}$ can be used to determine $\beta$ and hence the time delay in (1).

\section{UWB TIME-DELAY LINE}

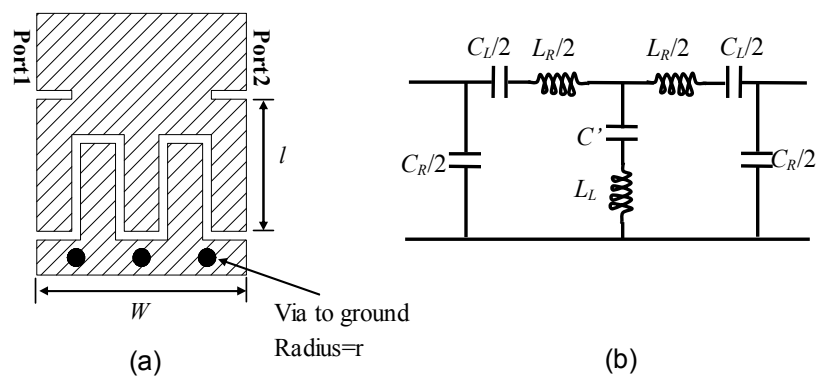

Figure 2 (a) Rotated structure and (b) equivalent circuit of the unit cell
Although (1)-(6) indicate that the structural parameters $L_{R}$, $C_{R}, L_{L}$ and $C_{L}$ can be used to provide a large value of $\beta$ for a large time delay, simulation studies have shown that a large $\beta$ would result in a narrow bandwidth. However, simulation studies also have shown that if the unit cell is rotated by 90 degree as shown in Fig. 2(a), the operation bandwidth would be much wider. Thus, for ultra-wideband operation, say, from 0.1-10 GHz, we propose to use the wideband unit cell in Fig. 2(a), with the equivalent circuit shown in Fig. 2(b). Noted that the wideband unit cell has a symmetrical structure. One of the main advantages of using such symmetrical structure is that we can use identical unit cells in cascade to provide a long time delay. Due to the symmetrical structure, the two S-parameters, $S_{11}$ and $S_{22}$, in each unit cell are equal and so there is no need to perform any matching process between the unit cells.

A periodic structure will have a slow-wave characteristic. Thus, to further increase the phase constant $\beta$, hence the time delay, of the unit cell, we use a periodic rectangular shape on the top edge of the unit cell as shown in Fig. 3, which shows a time-delay line employing a number of wideband unit cells in cascade.

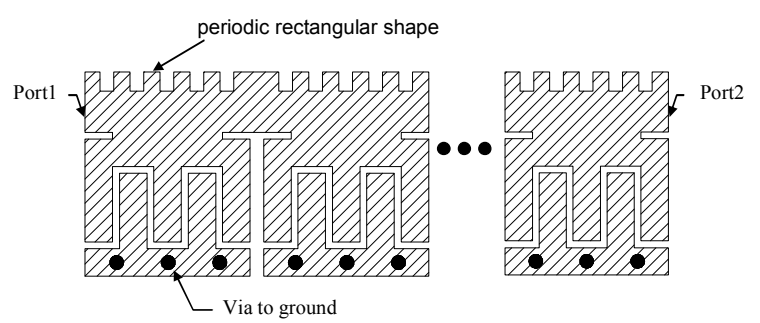

Figure 3 Time-delay line employing wideband unit cells in cascade

\section{SimUlATIONS, MEASUREMENTS AND DISCUSSIONS}

For illustration, a time-delay line employing 16 wideband unit cells in cascade, leading to a total length of $60 \mathrm{~mm}$, has been designed and optimized using the EM simulation tool CST MWS. In the optimization process, the number of fingers used in each unit cell was five as shown in Fig. 2(a) and the width of each finger was set to $0.2 \mathrm{~mm}$ (which is the smallest dimension we could make in our lab). The dimensions of the unit cell were optimized with the criteria of having (1) $\left|S_{11}\right|<$ $15 \mathrm{~dB}$, and (2) largest phase constant $\beta$. The final optimization results were used for fabrication on a F4B-2 PCB with a thickness of $0.8 \mathrm{~mm}$, permittivity of 2.65 and substrate tangent loss of $5 \times 10^{-4}$. For comparison, the time-delay lines using the PML and RH TL with the same length of $60 \mathrm{~mm}$ have also been designed, optimized and fabricated using the same substrate. The layouts and photographs of the three time-delay lines designed and fabricated are shown in Figs. 4 and 5.

Simulation studies have indicated that the length of the fingers has significant effects on $\left|S_{11}\right|$, so the optimization process mainly optimizes the length of the fingers. Simulation and measurement results on the two S-parameters, $\left|S_{11}\right|$ and $\left|S_{21}\right|$, of these time-delay lines are shown in Fig. 6 . The 
simulated and measured results on the time delays are shown in Fig. 7. It can be seen that, in all these studies, the simulated and measured results show good agreements. For the time-delay line using the wideband unit cells, Fig. 6(a) shows that the measured return loss is more than $15 \mathrm{~dB}$ (i.e., $\left|S_{11}\right|<-15 \mathrm{~dB}$ ) across the whole frequency band tested. Thus the impendence bandwidth is larger than from 0.1 to $10 \mathrm{GHz}$. The insertion loss is less than $1 \mathrm{~dB}$ (i.e., $\left|S_{21}\right|>-1 \mathrm{~dB}$ ) across the frequency band tested. The time delay achieved is 2000 ps across the whole frequency band tested, i.e. $0.1-10 \mathrm{GHz}$, as can be seen in Fig. 7.

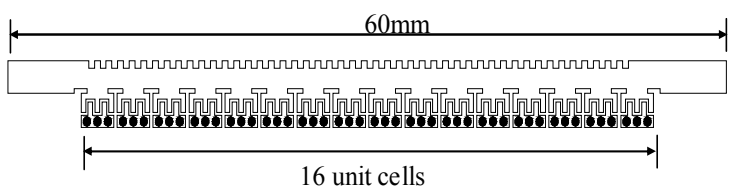

(a)

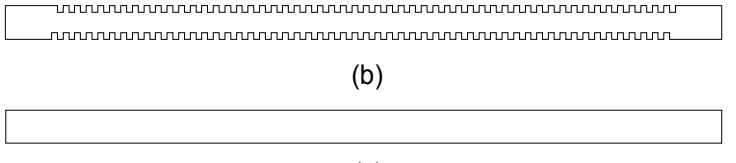

(c)

Figure 4 Layouts of time-delay lines using

(a) wideband unit cells, (b)PML and (c) RH TL

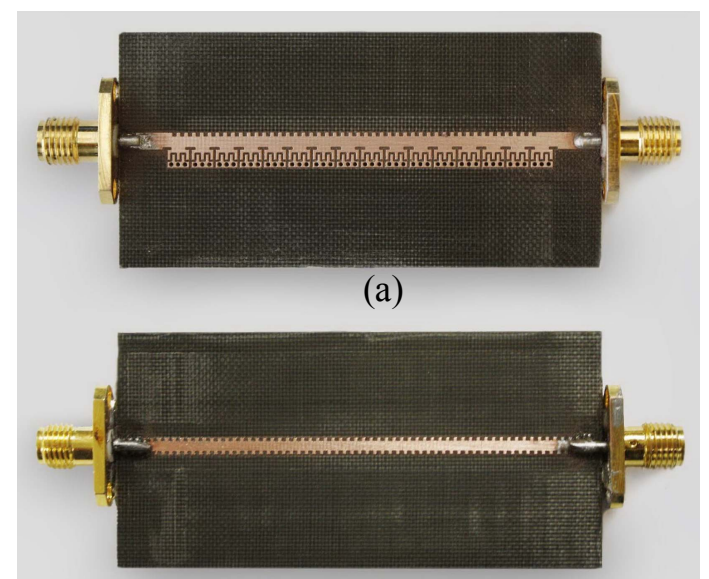

(b)

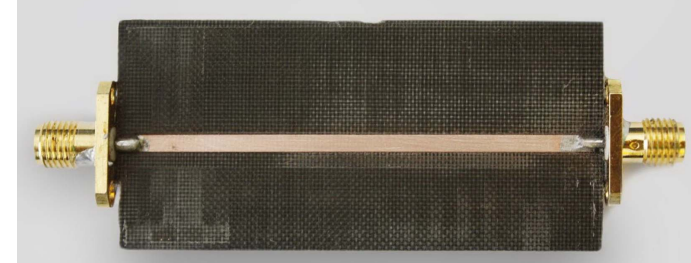

(c)

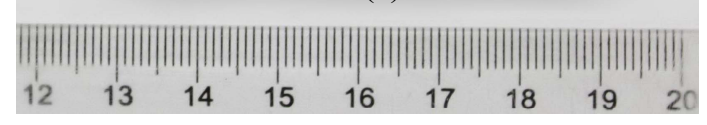

Figure 5 Photographs of time-delay lines using

(a) wideband unit cells, (b) PML, and (c) RH TL

The time-delay line using the PML also has quite a high return loss as shown in Fig. 6(b), although it is slightly lower than that of our proposed time-delay line. The insertion loss is also very low, less than $1 \mathrm{~dB}$. However, the time delay provided is less than $600 \mathrm{ps}$, substantially less than that of our proposed time-delay line. Figure 6(c) shows that the return loss of the time-delay line using the RH TL is even higher than that of our proposed time-delay line and the insertion loss is less than $1 \mathrm{~dB}$, but the time delay provided is only $300 \mathrm{ps}$.

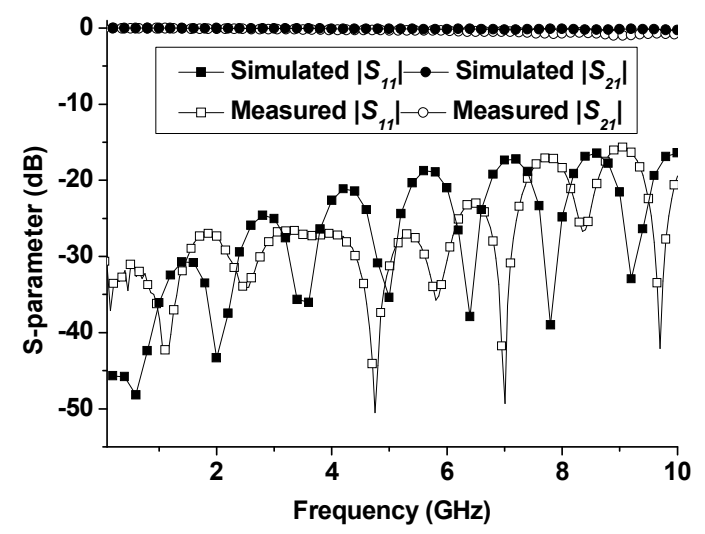

(a)

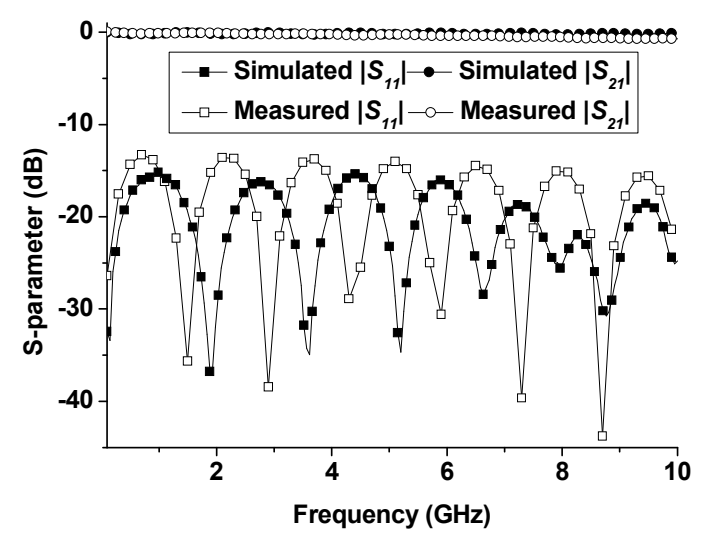

(b)

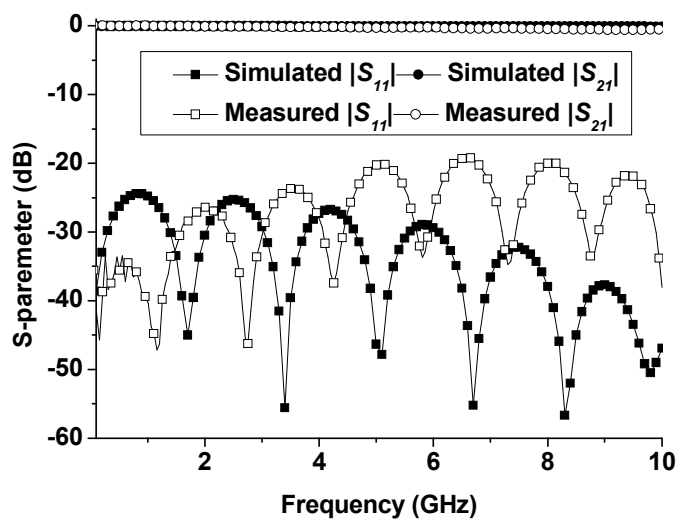

(c)

Figure 6 Simulated and measured $\left|S_{11}\right|$ and $\left|S_{2 l}\right|$ of time-delay lines using (a) wideband unit cells, (b) PML and (c) RH TL 


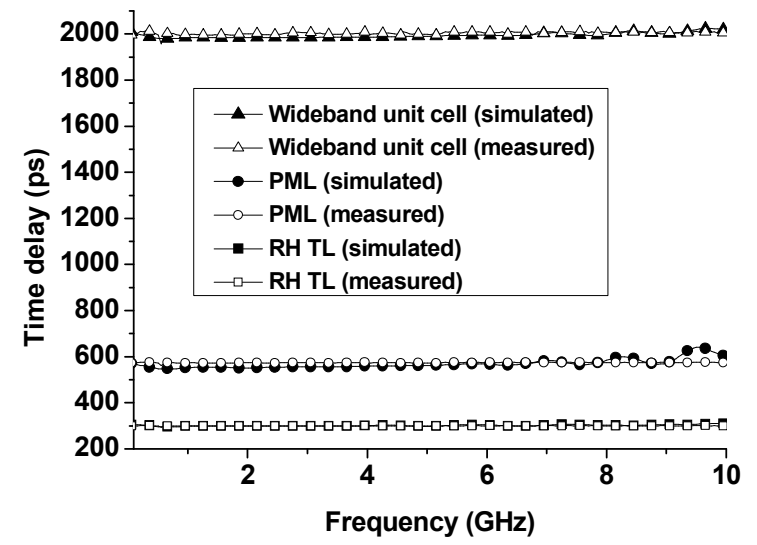

Figure 7 Simulated and measured time delays of different time-delay lines

\section{CONCLUSIONS}

A time-delay line based on using rotated versions of the conventional CRLH TL unit cells has been proposed, studied and fabricated. Two other time-delay lines using the PML and RH TL have also been designed and optimized for comparison. Simulation and measurement results have demonstrated that, for the length of $60 \mathrm{~mm}$, our proposed time-delay line has a much longer time delay of $2000 \mathrm{ps}$, more than 3 times and 6 times longer than that of the PML and RH TL, respectively, yet it has a wide operating bandwidth of $0.1-10 \mathrm{GHz}$, a high return loss and a low insertion loss.

\section{REFERENCES}

[1] P. B. Kenington, High-linearity $R F$ amplifier design, Artech House: Norwood, 2000, pp. 7-10.

[2] V. Veselago, "The electrodynamics of substances with simultaneously negative values of $\varepsilon$ and $\mu$," Soviet Physics Uspekhi, vol. 10, pp. 509-514, 1968.

[3] C. Caloz, H. Okabe, T. Iwai, and T. Itoh, "Transmission line approach of left-handed (LH) materials," Proc. USNC/URSI National Radio Science Meeting, vol. 1, pp. 39, San Antonio, Texas, June 2002,

[4] J. Zhang, Q. Zhu, Q. Jiang and S.J. Xu, "Design of time delay lines with periodic microstrip line and composite right/left-handed transmission line," Microwave and Optical Technology Letters, vol. 51, pp. 1679-1682, 2009.

[5] I. Bahl, Lumped elements for RF and microwave circuits, Artech House: Boston \& London, 2001

[6] E. G. Marc and A. P. Robert, "Modeling via hole grounds in Microstrip," IEEE Microwave and Guided Wave Letters, vol. 1, pp. 135-137, 1991.

[7] A. Lai, T. Itoh and C. Caloz, "Composite Right/Left-Handed Transmission Line Metamaterials," IEEE Microwave Magazine, vol. 5, pp. 34-50, 2004. 